\title{
A Versatile $2 \mathrm{MeV}, 200 \mathrm{~mA}$ Compact X-Band Linac
}

\author{
C. E. Clayton and K. A. Marsh \\ Department of Electrical Engineering \\ University of California at Los Angeles \\ 56-125B Engineering IV \\ Los Angeles, CA 90024
}

\section{Abstract}

A small, high performance electron linear accelerator is described. It is a modified version of a commercially available portable x-ray source. The fixed power supplies were replaced with remote controllable supplies allowing a systematic exploration the linac's parameter space. A condition in which the linac performs nearly in accordance with its theoretical limits was found. The $9.3 \mathrm{GHz} \mathrm{RF}$ linac and beamline deliver a $2 \mathrm{nsec}$ train of approximately 10 psec pulses with a peak current, limited by beam loading of the RF structure, of more than $200 \mathrm{~mA}$ and a beam energy of around $2 \mathrm{MeV}$ with a $5 \%$ FWHM energy spread. The small energy spread is due largely to the focusing of the beam through a $500 \mu \mathrm{m}$ lead pinhole early in the beamline. A $6 \mu \mathrm{m}$ Mylar vacuum window covers the pinhole allowing the use of the beam in a rough vacuum environment with minimal degradation of the beam emittance. The unnormalized beam emittance is $6 \pi \mathrm{mm}$-mrad and the final spot size is $260 \mu \mathrm{m}$ diameter for $f / 20$ focusing.

\section{INTRODUCTION}

A compact, reliable, inexpensive, and easy to operate electron linac was required for experiments on the plasma beat wave accelerator concept at UCLA. 1,2 Such a linac was realized through the modification of a commercially-available $\mathrm{x}$-radiography machine. This paper will briefly describe the hardware as well as some of the operational experience of the linac.

\section{ACCELERATOR HARDWARE}

\section{The Linac}

The electron linac started out as a commercial portable $x$ ray generator, the MINAC 1.5 (Refs. 3 and 4) manufactured by Schonberg Radiation Corporation 5 . The modifications we made to this unit are described in greater detail in Ref. 6 . Essentially, the modifications were the following. First, the tungsten target (the $\mathrm{x}$-ray source) at the end of the linac was removed and the linac attached to a vacuum system. The remainder of the modifications was essentially to replace the gun-voltage, grid-bias voltage, cathode-heater, and grid-pulser power supplies, which were not all independently adjustable in the commercial unit, with adjustable and remote-operable units. The $9.3 \mathrm{GHz}$ magnetron was also upgraded from a 200 to a $250 \mathrm{~kW}$ unit.

These changes allowed us to freely explore parameter space and fine-tune the operating parameters. The net result of these modifications was to increase the beam energy from $1.5 \mathrm{MeV}$ to $2 \mathrm{MeV}$ and the beam current from $2 \mathrm{~mA}$ to $25 \mathrm{~mA}$ average current.

\section{Vacuum system}

The pressure in the vacuum chamber at the interaction point in the beat wave experiment is typically $150 \mathrm{mT}$ of hydrogen gas. However, the pressure at the gun of the linac must be kept below $10^{-7} \mathrm{~T}$. One solution is to use differential pumping techniques to drop the pressure between the gun and the interaction point but this would have added a level of complexity that we could not afford. A simpler solution is to use a thin vacuum window to separate the rough vacuum of the experiment from the high vacuum of the linac. But at these low beam energies, even the thinnest foils adds a finite angular spread to the beam. However, if the foil is located at a tight waist, the degradation to the emittance is minimized.

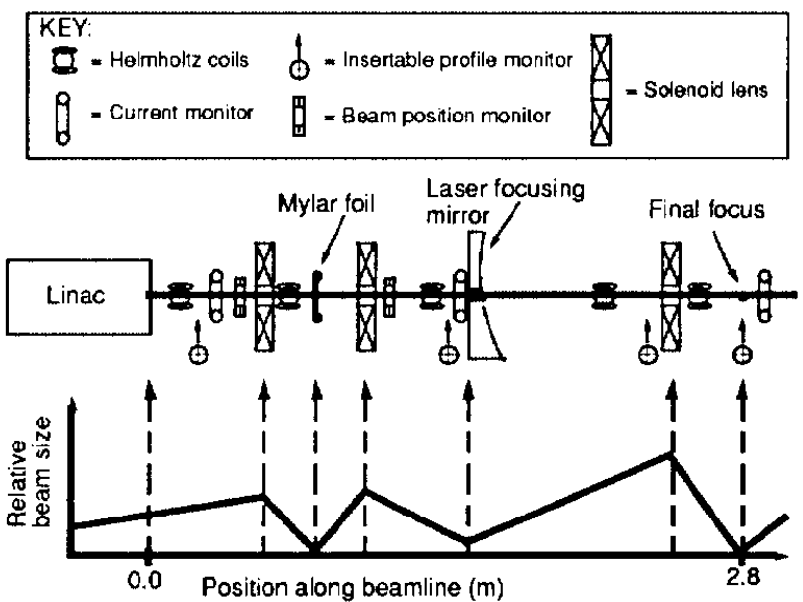

Figure 1: Cartoon of electron beamline and the variation of the beam spot size along the beamline.

Figure 1 shows the electron beam line with its three solenoidal lenses and three waists. A $6 \mu \mathrm{m}$ thick Mylar foil is located at the first waist. We find that the emittance increases from $4 \pi$ to $6 \pi \mathrm{mm}$-mrad in traversing the foil. For added mechanical strength, the foil is supported against the higher pressure downstream by a thin lead disk with a $500 \mu \mathrm{m}$, centrally located pinhole. The presence of the pinhole has the added benefit of selecting the momentum of the beam due to the chromatic aberration of the solenoid lens. Thus the fairly wide energy spread coming out of the linac is scraped down to a $5 \%$ spread after the pinhole.

\section{Beamline}

After the pinhole, the beam has two more waists, again from solenoid lenses. The beam line has five Helmholtz coils to keep the beam centered on the lenses and at the interaction 
point as well as various profile monitors and current monitors as shown in Fig. 1. Between the first two profile monitors,

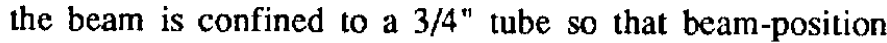
monitors are required to verify proper alignment of the beam.

The second waist in the beamline is at a small $(\approx 5 \mathrm{~mm})$ hole drilled in the final, off-axis-parabolic focusing mirror of the experiments large diameter $(15 \mathrm{~cm}) \mathrm{CO}_{2}$ laser beam. Beyond this point, the electrons are collinear with the laser beam, as is required for the experiment. At the last lens, the beam has a diameter of about $1 \mathrm{~cm}$ and is focused down to a spot of $260 \mu \mathrm{m}$ FWHM at a distance of about $20 \mathrm{~cm}$ from the lens.

\section{OPERATIONAL EXPERIENCE}

For all these measurements, the rf power is about $230 \mathrm{~kW}$, the gun voltage is $10 \mathrm{kV}$, the cathode temperature is well above the emission vs. temperature "knee", the grid bias is -90 $\mathrm{V}$ and the pulse amplitude applied to the grid is about $250 \mathrm{~V}$, well into saturation.

\section{Pulse width control}

As the width of the square pulse applied to the grid is varied from 10 to $20 \mathrm{nsec}$, the output current measured on the first current monitor rises from zero to about $100 \mathrm{~mA}$ with a $10 \mathrm{nsec}$ FWHM. For longer applied pulses, the current essentially droops with time. Energy measurements indicate that the longer pulses have severe energy droop as well. What is happening is that the first $10 \mathrm{nsec}$ of the output pulse substantially loads down the if fields, perhaps most strongly in the early cavities. For our experiment, we operate with a 15 or $20 \mathrm{nsec}$ drive pulse to stay just on the edge of severely loading down the rf fields and thus maximize extracted current

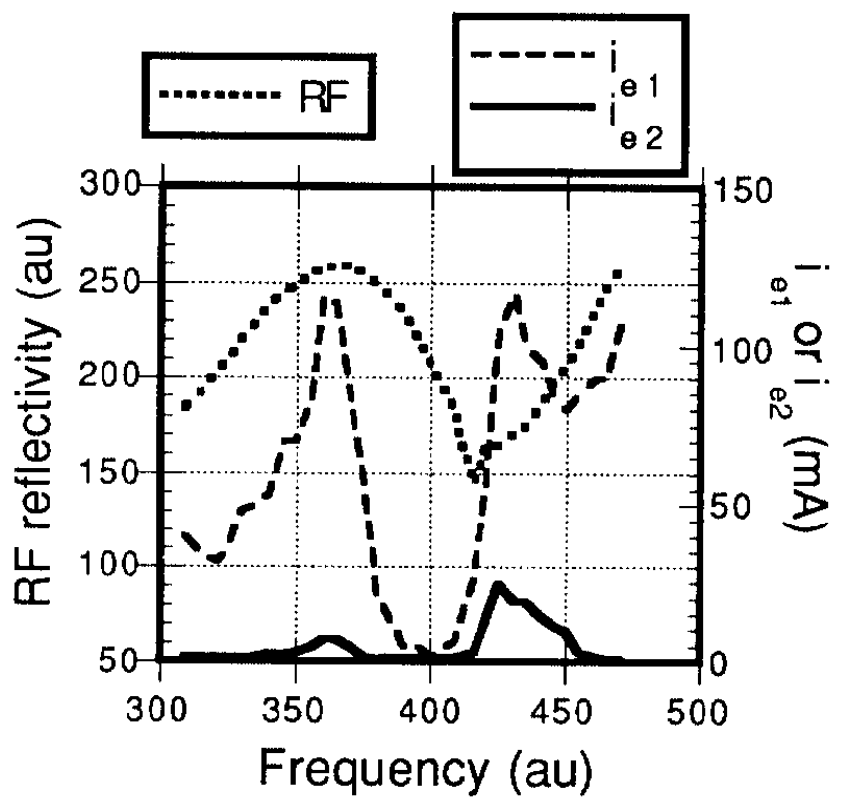

Figure 2: Tuning curves for the linac showing the variation of the reflected rf power and the beam currents measured before $\left(i_{\mathrm{e} 1}\right)$ and after $\left(\mathrm{i}_{\mathrm{e} 2}\right)$ the momentum-selecting lead pinhole.

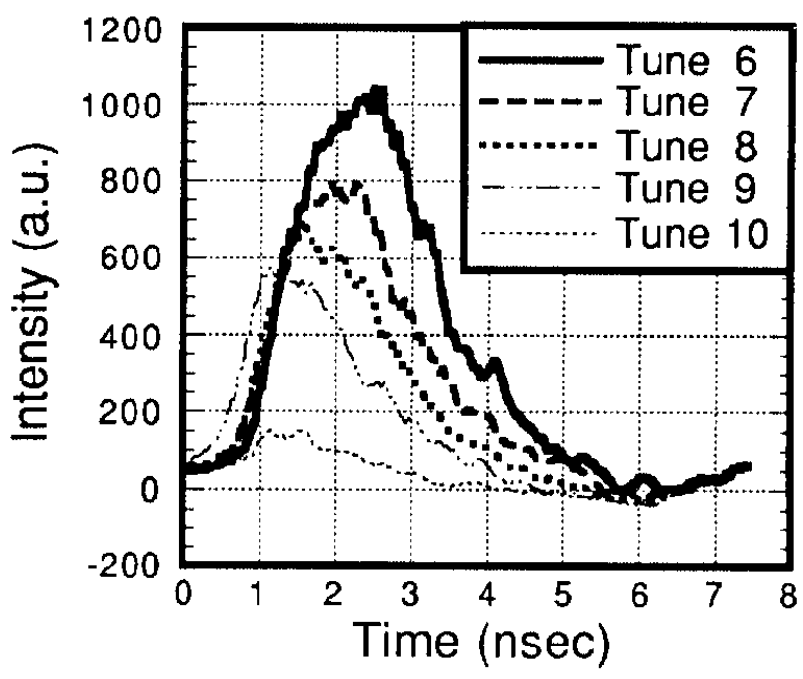

Figure 3: The output pulse shape measured at the interaction point for various frequency tunings of the magnetron.

while avoiding substantial energy droop on the beam.

\section{Tuning curve and macropulse structure}

The accelerating mode of the linac was designed to capture $10 \mathrm{keV}$ electrons and accelerate them to near light speed over the first third of the linac structure. As we increase the of frequency in the vacinity of the design frequency, we see a glitch in the curve of rf reflectivity vs. frequency (top curve in Fig. 2) as the accelerating mode becomes excited. At the same time, the signals on the first and second current monitors rise rapidly. The second monitor measures after the lead pinhole so essentially it measures the $2 \mathrm{MeV}$ portion of the beam whereas the first monitor sees all energies. As the frequency increases further, the second monitor falls off slowly as the rf reflectivity rises indicating that we are depositing less and less energy into the stored rf fields.

The output pulse shape at the interaction point was measurcd as a function of this tuning. Some of the results are shown in Fig. 3. The top curve labeled "Tune 6" is at the optimum frequency from Fig. 2. As the frequency was increased up to this optimum (Tunes 1 through 5 , not shown) the pulse shape was constant, only the amplitude changed. This may be due to the poor capture efficiency in the early cavities at frequencies below the optimum. But for higher frequencies (Tunes 7 through 10 in Fig. 3), the pulse shape changes as the rear of the pulse is lost due to a saturation in the current at progressively lower levels. This is the slow drop seen in Fig. 2 and is probably due to beam loading since we store less and less energy in the accelerating mode as we move up in rf reflectivity.

\section{Micropulse structure}

The micropulse structure was measured with a Cherenkov emitter located at the interaction point. The emitter was a short piece of $1 \mathrm{~mm}$ diam quartz fiber optic rotated such that the beam entered at $47^{\circ}$ to the fiber axis. In this case, a 
portion of the Cherenkov cone is directed along the fiber axis. This light was relayed with lenses to an optical streak camera and the signal was recorded at several different sweep speeds. Figure 4 shows the entire micropulse with a time resolution of about $20 \mathrm{psec}$. The variation from one micropulse to the next is dominated by the streak camera photoelectron statistics. (The signal was intentionally kept weak for optimal time resolution). A single micropulse from a faster streak is shown in Fig. 5. The time resolution for this streak was about 6 psec which implies the true pulse width is around $10 \mathrm{psec}$ FWHM. The current monitors measure an average current of $>20 \mathrm{~mA}$ which means that the peak current in one micropulse is in excess of $200 \mathrm{~mA}$ at $2 \mathrm{MeV}$.

\section{SUMMARY}

In summary, a linac with the interaction-point parameters of: $2 \mathrm{MeV}$ energy, $10 \mathrm{psec} / 200 \mathrm{~mA}$ micropulses, $2 \mathrm{~ns} / 20 \mathrm{~mA}$ macropulses, $6 \pi \mathrm{mm}$-mrad emittance, $260 \mu \mathrm{m}$ FWHM spot

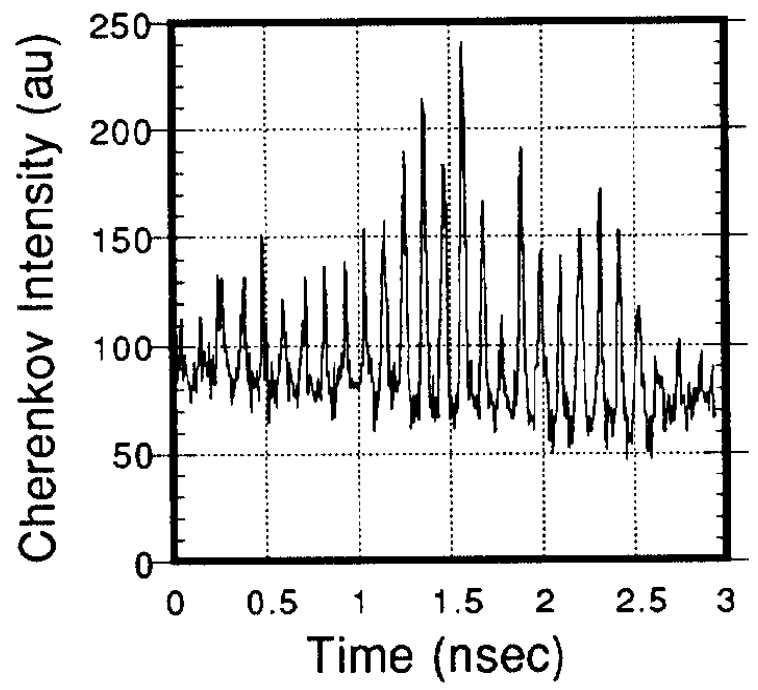

Figure 4: Lineout of a streak camera record of the Cherenkov emitter intensity (proportional to instantaneous beam current) vs. time for a slow sweep ( $20 \mathrm{psec}$ resolution).

size, and single shot (but capable of high repetition rates) was built by modifying a commercial $\mathrm{x}$-ray source. The beam can be delivered to a course vacuum environment. The linac is reliable and easy to operate and can be switched on, tuned up, and characterized in under 30 minutes.

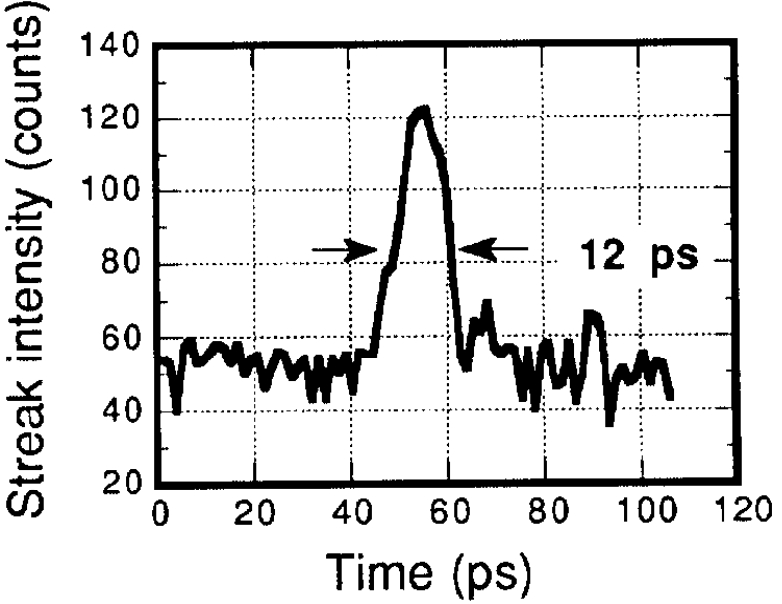

Figure 5: Lineout of a streak camera record of the Cherenkov emitter intensity (proportional to instantaneous beam current) vs. time for a fast sweep ( $6 \mathrm{psec}$ resolution). The deconvolved pulse width is closer to 10 psec.

\section{ACKNOWLEDGMENTS}

The authors would like to thank Dr. R. G. Schonberg, Dr. R. H. Miller, and Dr. W. A. Johnson for many useful discussions. We also thank $M$. Everett for the streak measurements. This work is supported by the U. S. Department of Energy under grant no. DE-FG03-92ER40727.

\section{REFERENCES}

1 C. E. Clayton, K. A. Marsh, A. Dyson, M. Everett, A. Lal, W. P. Leemans, R. Williams, and C. Joshi, "Ultrahigh-gradient acceleration of injected electrons by laser-excited relativistic electron plasma waves", Phys. Rev. Lett. 70, 37 (1993).

2 C. E. Clayton, K. A. Marsh, M. Everett, A. Lal, and C. Joshi, "Demonstration of Plasma Beat Wave Acceleration of Electrons from $2 \mathrm{MeV}$ to $20 \mathrm{MeV}$ ", this proceedings.

3 R. H. Miller, H. Deruyter, W. R. Fowkes, J. M. Potter, R. G. Schonberg, and J. N. Weaver, "RF phase focusing in portable $x$-band, linear accelerators", IEEE Trans. Nuc. Sci. NS-32, 3231 (1985).

4 R. G. Schonberg, H. Deruyter, W. R. Fowkes, W. A. Johnson, R. H. Miller, J. M. Potter, and J. N. Weaver, "Portable, xband, linear accelerator systems", IEEE Trans. Nuc. Sci. NS. 32, 3234 (1985).

5 Schonberg Radiation Corp., Mountain View, CA.

6 C. E. Clayton and K. A. Marsh, "A $2 \mathrm{MeV}, 100 \mathrm{~mA}$ electron accelerator for a small laboratory environment", Rev. Sci. Instr., 64, 728 (1993). 